Journal of Medical and Health Studies (JMHS)

ISSN: 2710-1452

DOI: 10.32996/jmhs

Journal Homepage: www.al-kindipublisher.com/index.php/jmhs

\title{
The Stroke Knowledge Assessment Tool (SKAT): Development, Reliability and Validity
}

\author{
Reuben Grech ${ }^{1}$ and Paulann Grech ${ }^{2} \square$ \\ ${ }^{1}$ Medical Imaging Department, Mater Dei Hospital, Malta \\ ${ }^{2}$ Department of Mental Health, Faculty of Health Sciences, University of Malta, Msida, Malta
}

$\square$ Corresponding Author: Paulann Grech, E-mail: paulann.grech@um.edu.mt

ARTICLE INFORMATION

Received: 08 September 2021

Accepted: 14 October 2021

Published: 19 October 2021

DOI: 10.32996/jmhs.2021.2.2.9

\section{KEYWORDS}

Stroke Knowledge, Assessment

tool, Stroke, Instrument

development, Stroke awareness

\section{ABSTRACT}

The purpose of the study was to develop and test the psychometric properties of a stroke knowledge assessment tool based on a mixed-methods approach and a synthesis of the relevant literature on stroke. The content of the tool was predominantly developed by the findings from a qualitative study involving ten stroke patients who had been admitted to a state hospital due to a stroke during the previous 2 years. The other source for tool construction was the information that emerged from an extensive literature review. The resulting online questionnaire was divided into three main sections namely i) demographics ii) stroke knowledge and iii) stroke response. A 5-point Likert scale was chosen to answer each question in Sections B and $C$, which facilitates the analysis process using descriptive and inference statistics. The tool was subjected to psychometric testing with promising results. The use of a standard tool, such as SKAT, which is the one presented in this paper, presents health professionals, who are in a prime position to gauge healthcare educational needs, with the means to assess stroke knowledge and carry out interesting uniform comparisons between different geographical and ethnical populations. The targeted audience for the use of this tool is a). The general population and/or b). A population where stroke knowledge enhancement is being planned. SKAT can then inform educational and awareness needs.

\section{Introduction}

One of the significant causes of disability and mortality all over the world is stroke. It has such severe consequences that $37 \%$ of sufferers die during the first year of having experienced it (Thrift, Dewey, Macdonell, McNeil, \& Donnan, 2000). As medicine progressed, a range of treatment options have become available yet many of these can only offer a degree of effectiveness if the patient presents to medical services in a timely manner. Literature on the topic shows that timely presentation for treatment is highly linked to knowledge about stroke, especially its symptomatology and awareness of the required actions during onset. Thus, stroke knowledge assessment is crucial to the understanding of certain aspects of acute stroke, including the public's preparedness, response, and outcomes. Such assessments should form the basis of national stroke campaigns in order to increase their costeffectiveness. According to Hou et al. (2017): "Accurate assessment of stroke knowledge (SK) is fundamental to the successful understanding of, monitoring of, and intervening to improve the SK of patients and the public". Improving such knowledge may indeed reduce the prevalence of primary and secondary stroke (Inzitari et al., 2000) as well as improve long-term stroke clinical outcomes. Whilst some of the aspects related to stroke knowledge can be accessed easily, other biopsychosocial factors associated with this condition can only be thoroughly explored through a holistic and comprehensive assessment. Some of these factors that contribute to the complexity of stroke shall be described in the next section.

\section{Literature review}

\subsection{Stroke Knowledge}

Many factors have a role in an individual's total understanding of stroke and its management, according to the literature on stroke knowledge.

Copyright: (C) 2021 the Author(s). This article is an open access article distributed under the terms and conditions of the Creative Commons Attribution (CC-BY) 4.0 license (https://creativecommons.org/licenses/by/4.0/). Published by Al-Kindi Centre for Research and Development, London, United Kingdom. 
Gender is one factor that has been found to affect stroke knowledge. Marx et al. (2010) carried out research in Western Germany to investigate gender-specific differences in stroke awareness and found that $71.3 \%$ of females could identify at least one warning sign of stroke whilst males could do so less successfully (57.8\% - p 0.01). When asked to provide the emergency services number, females were also better at this ( $33.3 \%$ vs $24.3 \%$, p 0.05) and when asked about the actions to be taken during an acute stroke, females also outperformed males (87.2\% vs $70.1 \%$, p 0.001). Yoon et al. (2001) reported similar findings in a separate study.

Age has been considered as a variable in a series of studies dedicated to stroke knowledge. In a systematic review that consisted of 39 trials, Jones et al. (2010) found that $40 \%$ of participants who were 45-64 years old could mention two-stroke symptoms whilst only $32 \%$ of those over 65 years old could do so. There was also a difference in knowledge of stroke risk factors between the two groups. In contrast to $30 \%$ of participants over the age of $65,50 \%$ of patients under the age of 65 were aware of their potential risk factors. In their studies, Yoon et al. (2001) and Hickey et al. (2001) obtained similar findings. Older age has also been related to a lower probability of calling emergency services when a stroke occurs (Jurkowski, Maniccia, Dennison, Samuels, \& Spicer, 2008). Stroke awareness campaigns aimed at the elderly, on the other hand, have been proven to be ineffective (Reeves, Rafferty, Aranha, \& Theisen, 2008; Silver, Rubini, Black, \& Hodgson, 2003). This is problematic since advanced age is linked to an increased risk of stroke.

Another factor linked to stroke knowledge is being born into or living in a certain cultural environment for an extended period of time. Ethnic minorities have the greatest incidence of stroke and are also more prone to suffer severe side effects (Horner, Matchar, Divine, \& Feussner, 1991; Jones et al., 2000; Shen, Washington, \& Aponte-Soto, 2004). According to other studies, ethnic minorities have reduced awareness of stroke warning signs and are less likely to take the required actions when a stroke occurs (DuBard, Garrett, \& Gizlice, 2006; Schneider et al., 2003).

Finally, poor literacy has been shown to obstruct access to health care, and this is not exclusive to stroke patients (Lasater, Davidson, Steiner, \& Mehler, 2001; Wilson, Chen, Grumbach, Wang, \& Fernandez, 2005). Furthermore, individuals with literacy difficulties will find it more difficult to participate in stroke awareness campaigns, which often utilize written material (Williams, Baker, Parker, \& Nurss, 1998). It is also conceivable that people with low literacy may feel humiliated during medical visits (Baker et al., 1996). When healthcare professionals fail to identify literacy problems, these individuals' negative emotions are often reinforced (Parikh, Parker, Nurss, Baker, \& Williams, 1996). This causes anxiety and a lack of trust in some people, causing them to postpone the act of seeking medical assistance (Lee, 1999).

\subsection{Stroke Knowledge and its assessment}

At present, there is no consensus on the definition of stroke knowledge, which may refer to an individual's understanding of the aetiology, risk factors, symptomatol-ogy, emergency response, treatment, and/or outcomes of stroke. This had led to different authors addressing different aspects of stroke knowledge in their research. Though the latter may be appropriate to a specific study, it makes temporal and longitudinal (between different countries) comparisons difficult or even impossible. Since all of the aspects mentioned above are important, it is probably best to include all of them in a more holistic definition of stroke knowledge.

Assessing stroke knowledge is therefore not a straightforward task. If pre-existing tools are to be used, one should ensure that these have been developed using a comprehensive conceptual framework and that quality tests for reliability, validity, and feasibility (Jordan, Osborne, \& Buchbinder, 2011) have been performed to assess the tool's quality and performance.

Hou et al. (2017) performed a systematic review to identify and appraise tools for assessing stroke knowledge. Their appraisal checklist was based on Jordon's criteria (Jordan et al., 2011), the Consensus-Based Standards for the Selection of Health Measurement Instruments (Mokkink et al., 2010), and items of Health Technology Assessment reports (Fitzpatrick, Davey, Buxton, \& Jones, 1998). Fifty-nine stroke knowledge tests were included in their analysis, 49 (83.1\%) of which were used only in a single publication. The remaining ten tests were used to assess the knowledge of either the public (7 studies) or stroke patients ( 3 studies). The BRFSS heart and stroke module (Centers for Disease Control and Prevention, 2011) was the most widely used test to assess stroke knowledge, although it appears to not have been validated and assesses only a few stroke knowledge concepts.

The Stroke Knowledge Test (SKT) (Sullivan \& Dunton, 2004), assessing different aspects of stroke knowledge, was found to be the most comprehensive test. Although the content validity, discriminative validity, and face validity of the Stroke Action Test (STAT) have been sufficiently evaluated (O'Mahony, Rodgers, Thomson, Dobson, \& James, 1997), the test is not based on a conceptual framework (Billings-Gagliardi \& Mazor, 2005). Hou et al. (2017) found that only the SKT and STAT fulfilled all their feasibility criteria. Out of the included studies, Hou et al. (2017) found that only the SKT "was developed using rigorous methodology, covers a wide range of concepts, fits all feasibility criteria, and has validated psychometric properties". However, the SKT was not developed using a specific conceptual framework and the validation of its psychometric properties is not exhaustive. The authors, therefore, concluded that "no current SK test can be highly recommended for clinical and for research use" (Hou et al., 2017). 
The other major limitation of utilizing the existing tools is the fact that they do not consider the local cultural, social, genetic, and demographic settings in which studies are carried out, which may be crucial to the understanding of aetiology and epidemiology of stroke.

With this in mind, and with the intention of being as comprehensive as possible when assessing stroke knowledge, the authors decided to develop a novel (stroke knowledge assessment) tool based on a mixed-methods approach and a synthesis of the relevant literature on stroke. The targeted audience for the use of this tool is a). The general population and/or b). A population where stroke knowledge enhancement is being planned - here the tool will help to identify the exact areas that education needs to focus on e.g.more knowledge about the action to be taken when subjected to a stroke.

\section{Methodology}

Aim: The purpose of the study was to develop and test the psychometric properties of a stroke knowledge assessment tool (SKAT) based on a mixed-methods approach and a synthesis of the relevant literature on stroke.

\subsection{Method}

The content of the tool was predominantly developed by the findings from a qualitative study involving ten stroke patients who had been admitted to a state hospital due to a stroke during the previous 2 years (to allow for accurate memory recall) but not earlier than 3 months prior to data collection (to allow for recovery following the acute period). Following the necessary ethical clearances, a purposeful random sampling technique was used to select ten participants. A semi-structured interview was carried out with each of the participants, for which the patient could also invite a by-stander, which experienced the onset of stroke. Ethics permission was provided by the University's Research Ethics Committee (Reference no. 012704) and all participants provided written informed consent. The thematic analysis of the data that emerged from the interview was carried out as guided by Braun and Clarke (2006). In particular, this involved a process during which selected quotes led to the extraction of codes which were then grouped into overarching themes.

The other source for tool construction was the information that emerged from an extensive literature review. An extensive electronic search was performed using 'StarPlus - Library Catalogue' and the Ovid MEDLINE database without language restrictions for studies published in the past 10 years. To enhance the sensitivity of the search, free text and subject headings (MeSH and EMTREE) were utilized. Four main keywords were derived from the aims of this research: 'stroke knowledge', 'stroke recognition', 'stroke education', and 'stroke preparedness'. Exploded headings relating to these keywords were combined with the Boolean operators 'OR' and 'AND' for use in the searches. Synonyms and wildcards were utilized to expand the search and increase its sensitivity. In order to validate the search strategy, manual searching was next performed through the two journals that contributed the largest number of papers to this review, Stroke: A Journal of Cerebral Circulation and the International Journal of Stroke. Titles and abstracts of retrieved studies were initially screened to identify eligible studies for inclusion. Full-text articles were then obtained for these studies as well as in cases where the primary evaluation was not sufficient to determine whether a study should be included or not. Any inclusion and exclusion decisions during this stage were documented and used to complete a Preferred Reporting Items for Systematic Review and Meta-analysis (PRISMA) flowchart (Moher, Liberati, Tetzlaff, and Altman (2009)). Articles that were not in the English language were translated. Reference management software (EndNote X7) was used to organize the articles retrieved and to exclude duplicate publications.

In order to facilitate and portray the conversion of qualitative data to quantitative survey questions, a framework was constructed (Grech \& Grech, 2021). This ensured that all-important qualitative themes were addressed in the quantitative stage, a necessary process because neglecting to address the comprehensive interchange of data between the stages is often a major pitfall of mixed methods approaches. Moreover, the development and use of such a framework were also aimed at enhancing transparency. A template of the framework used is shown in Figure 1. 


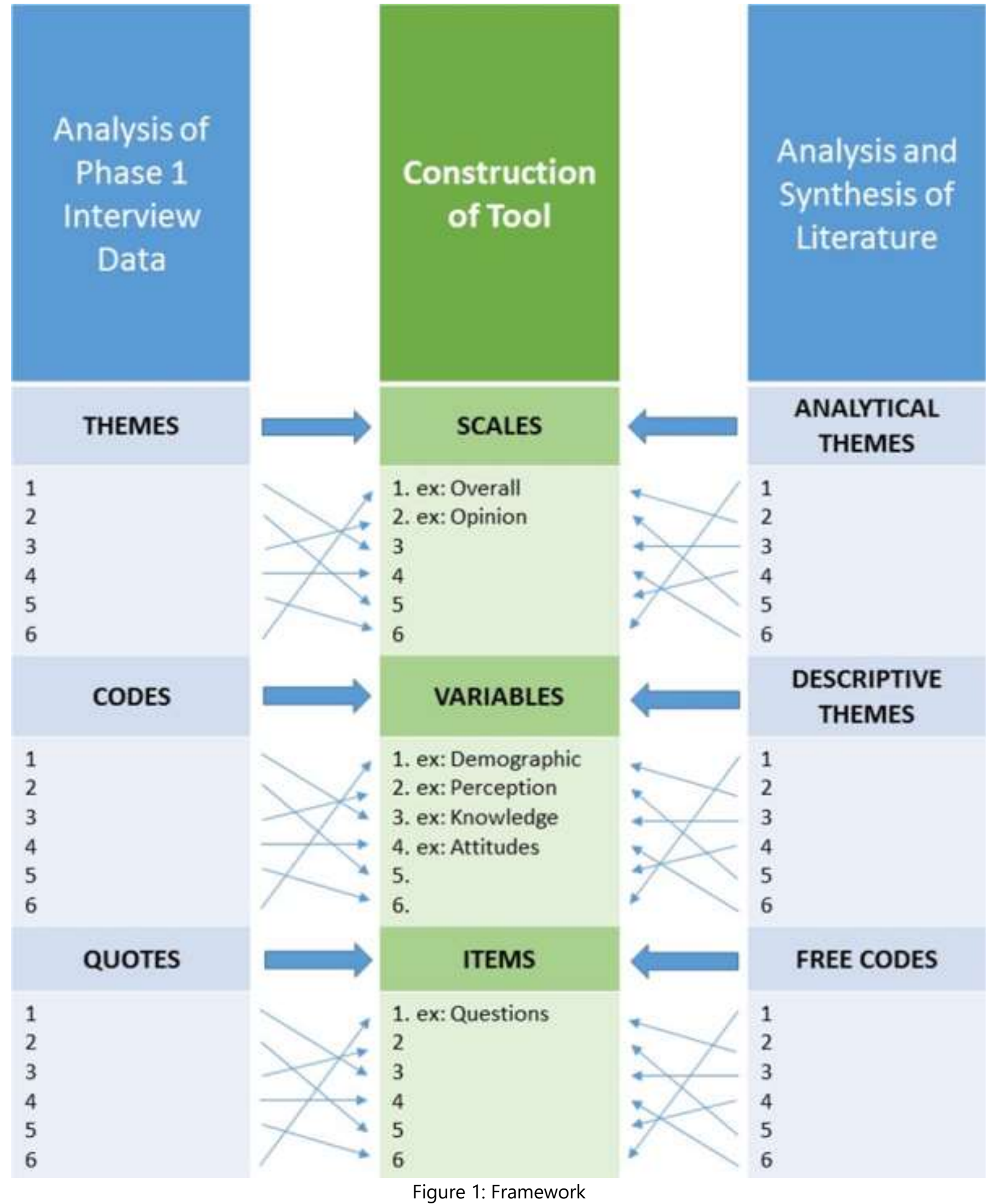

\subsection{Instrument}

The framework was constructed based on the guidance offered separately by Braun and Clarke (2006), Thomas and Harden (2008), DeVellis (2012), Creswell (2015), and Tay and Jebb (2017). In his guidance on tool construction, Creswell (2015) described the process of translating qualitative results into a quantitative instrument. To this extent, he referred to qualitative results, which have already been analyzed thematically into quotes, codes, and themes. At this stage, he recommended the conversion of quotes into the new instrument's items, the codes into variables, and the themes into scales. This guidance was followed during the construction of the instrument in the current study. However, rather than using the interview results only in the construction of the tool, it was decided to also include certain aspects from the existing body of the literature on the subject. The reason behind using 
both of these sources was to construct a tool that reflects both the patient's experiences but which is also informed by the existing evidence base on the topic.

In an article by Thomas and Harden (2008), the authors presented a process of thematic analysis as applied to a body of literature rather than to raw qualitative data as per the norm. The rationale for this application was to explore and promote the use of a more structured and rigorous process when attempting to integrate the results of qualitative studies in systematic reviews. The authors exhibited the use of such an approach, which they termed 'thematic synthesis', in a group of qualitative studies on health promotion research. The guidance on the process of thematic analysis offered by Braun and Clarke (2006) was followed. However, instead of selecting quotes for coding, the authors reviewed the results of each qualitative study and highlighted the main points which they described as 'free codes' rather than quotes. The free codes were subsequently grouped into 'descriptive themes' which were further reduced to 'analytical themes'. While the descriptive themes were essentially a summary of the results of the primary studies, the analytical themes went a step further by providing an element of interpretation in an addition to grouping the descriptive themes. This approach enabled the generation of novel constructs, explanations, and hypotheses(Thomas \& Harden, 2008). In the current study, the thematic synthesis process described by Thomas and Harden (2008) was applied to the integration of the studies included in the literature review. This process allowed for the transparent integration of the literature into the new tool.

The outcome of the parallel analysis of the interview data and the literature was next used to inform the construction of the tool (Figure 1). Thus, quotes (column 1) and free codes (column 3) became items within the tool (column 2). Similarly, codes (column 1) and descriptive themes (column 3) were translated into variables (column 2). Finally, themes (column 1) and analytical themes (column 3) were used to form scales (column 2). This part of the process of the construction of the tool was informed by Creswell (2015).

The questionnaire was divided into three main sections namely i) demographics ii) stroke knowledge and iii) stroke response. A 5point Likert scale was chosen to answer each question in Sections B and C, which facilitates the analysis process using descriptive and inference statistics.

The demographics section included the following variables: gender, nationality, ethnicity, education, income, risk factors, and whether the participant was a health care worker. As discussed below, these variables were chosen following an extensive literature review and are meant to be universal and applicable in different settings.

The Knowledge Section comprises 17 questions, which explore the following aspects of stroke: symptoms, risk factors, aetiology, and stroke campaigns. Similarly, the Response section included 17 questions that explored stroke response, the reasons underlying such response, and stroke treatment.

Since the authors were interested in a tool that could reliably and comprehensively assess stroke knowledge in a large population (e.g.. for use in national epidemiological studies or stroke campaigns) it was decided that an online survey would represent the ideal platform for such a tool. Please refer to Appendix 1 for the tool.

\section{Results and Discussion}

Following its construction, the tool was subjected to a number of tests in order to assure its quality. This was primarily done using a critical appraisal framework developed by Hou et al. (2017) in their endeavour to assess the quality of stroke knowledge assessment tools included in their systematic review. The authors developed this framework by consulting with professionals and by synthesizing three already-established criteria and checklists. The resulting appraisal framework consisted of three sections: (1) conceptual framework, (2) psychometric properties (e.g. validity and reliability), and (3) feasibility (Hou et al., 2017). These three sections of the critical appraisal framework were applied to the stroke knowledge assessment tool developed in the current study, as described below.

\subsection{Conceptual framework}

As described in the Method section, the tool was developed using existing evidence on the area of interest as well as semistructured interviews with stroke patients. Thus, it seems acceptable to state that the tool has a sound conceptual framework.

\subsection{Psychometric properties}

\subsection{Validity testing}

To ensure face validity, the questionnaire was scrutinized by a panel of experts in the field of stroke and later discussed within a focus group on the subject consisting of six laypersons. The experts were specifically asked to assess the questionnaire in terms of the (i) clarity of wording, (ii) style and formatting, (iii) feasibility, and (iv) readability (Haladyna, 2004; Trochim, 2001). They were 
also asked whether they thought the questionnaire could adequately assess stroke knowledge and to provide feedback that could enhance the representativeness of the instrument. Their experience and judgment were crucial assets here and helped to identify potential flaws prior to starting data collection. The effect of literacy on reading comprehension was subsequently assessed within the focus group, and there was good agreement between the two samples (experts and lay persons). Since the panel of stroke experts was familiar with the construct of interest, they were also asked to assess the degree of relevancy and representativeness of each item within the questionnaire (Haynes, Richard, \& Kubany, 1995). The outcomes are summarised in Table 1.

Table 1 Appraisal Criteria for Validity [adapted from Hou et al. (2017)

\begin{tabular}{|l|l|l|l|}
\hline & \multicolumn{2}{|l|}{} & $\begin{array}{l}\text { Consensus } \\
\text { outcome }\end{array}$ \\
\hline i) & Face validity & Does the test appear to assess what it is supposed to? & Yes \\
\hline ii) & Content validity & Are intended concepts clearly stated? & Yes \\
\hline
\end{tabular}

\subsection{2 - Reliability testing}

The new instrument was converted into an online version and uploaded to an online platform. In order to explore the reliability of the instrument, a pilot study was carried out. The pilot study was then employed and involved data collection on two instances from a sample of 308 individuals with similar characteristics to the actual study sample. Individuals who participated in the pilot study were provided with a three-digit code to allow the comparison of answers from one point to another while safeguarding anonymity. One of the challenges faced was the dilemma between allowing for too large a time-lapse between one data collection point and another, thus risking the answers being affected by intervening circumstances and, conversely, retesting immediately with the risk of the participants recalling the answers given during the first session (Polit \& Beck, 2010). As advised by Parahoo (2014), a minimum time lapse of 2 weeks is needed to prevent the recall of material by participants. Thus, the authors opted to allow for a 1-month period prior to retesting. Consequently, the following reliability tests were conducted:

\subsubsection{Test-retest (stability) reliability}

The Statistical Package for Social Sciences (SPSS) ${ }^{\circledR}$ (Version 24.0) was used to assess the reliability of the tool. This was achieved by counting the participants' answers separately for each of the two data collection points and analyzing them using the intraclass correlation (ICC) test. The results obtained are shown in Table 2 . The intra-class correlation co-efficient was found to be 0.79 which is considered as 'excellent' according to Cicchetti's guidelines for interpreting ICC (Cicchetti, 1994).

Table 2 Intra-class correlation table

\begin{tabular}{|l|l|l|l|}
\hline $\begin{array}{l}\text { Number of } \\
\text { participants }\end{array}$ & $\begin{array}{l}\text { Time lapse between } \\
\text { the two data } \\
\text { collection points }\end{array}$ & $\begin{array}{l}\text { Method used for } \\
\text { identification of } \\
\text { participants }\end{array}$ & $\begin{array}{l}\text { Intra-class } \\
\text { correlation } \\
\text { coefficient }\end{array}$ \\
\hline $\begin{array}{l}\mathbf{1 1 8} \text { (out of } 308 \text { who } \\
\text { participated in the } \\
\text { pilot study) }\end{array}$ & 30 days & 3-digit code & $\mathbf{0 . 7 9}$ \\
\hline
\end{tabular}

\subsubsection{Internal consistency}

Internal consistency was assessed in SPSS by measuring Cronbach's alpha, which ranges from a result of 0 (no correlation between items) to 1 (perfect correlation). The reliability analysis of the tool yielded a Cronbach's alpha of 0.832 for the Stroke Knowledge section and 0.775 for the Response section.

\subsection{Feasibility}

In addition to the tests described, the conduction of the pilot study allowed other issues related to the tool to be explored, such as its feasibility, ease of comprehension, and other practical matters. Thus, following the pilot study, a focus group that included six of the participants was carried out to obtain feedback on these issues. The results of the feasibility testing can be viewed in Table 3. 
Table 3 Feasibility table

\begin{tabular}{|l|l|l|l|l|}
\hline \multicolumn{2}{|c|}{} & n (\%) & Unsure & No \\
\cline { 3 - 5 } \multicolumn{2}{|l|}{} & Yes & $1(16.7)$ & 0 \\
\hline 1 & Time and effort needed & $5(83.3)$ & $1(16.7)$ & $1(16.7)$ \\
\hline 2 & Ease of scoring & $4(66.7)$ & 0 & $1(16.7)$ \\
\hline 3 & Number of items & $5(83.3)$ & 0 & 0 \\
\hline 4 & Accessibility & $6(100)$ & & \\
\hline
\end{tabular}

\subsection{Implications for practice}

Because stroke knowledge is characterized by a variety of factors, it may be difficult for professionals to evaluate and incorporate all of the relevant aspects while evaluating knowledge. SKAT, which was constructed by a thematic synthesis of current research on the subject and a grass-roots population approach, maybe a helpful tool for exploring knowledge by taking into account the key variables in this field. Because the instrument was built in part based on a synthesis of research studies performed in various cultural contexts, it has the additional strength of taking into account and including cultural (and other) factors that are essential in stroke under-standing and evaluation. This emphasizes the tool's adaptability to various cultures.

Although SKAT was created as an online tool, the medium of presentation may simply be changed to a printed questionnaire or used in an interview-style format. This may increase the tool's adaptability and accessibility to a wider range of people.

\subsection{Limitations}

During the testing of the tool, an online format was used. This meant that it could be accessed only by computer-literate individuals and who had internet access. This may have resulted in selection bias. The researcher has been actively involved in the area of study for a number of years. This may have lead to certain pre-existing beliefs or hypotheses which may have unintentionally affected the interpretation of the data. In order to try to limit this effect, the researcher sought to continuously re-evaluate his impressions of participants and challenge any pre-existing assumptions throughout the study.

\section{Conclusion}

Following an extensive literature review, it was immediately evident that the majority of stroke knowledge questionnaires have been developed for the purpose of a particular trial and were rarely used in other populations. To this extent, consideration of cultural, social, genetic, and demographic settings was limited, which may pose challenges for understanding the aetiology and epidemiology of stroke. This led to the conclusion that "no current SK test can be highly recommended for clinical and for research use" (Hou et al., 2017).

The proposed tool in this current study, SKAT, was informed by in-depth qualitative interviews with stroke patients and a review of the relevant literature in order to ensure its comprehensiveness. The tool was also extensively validated prior to its use.

The use of a standard tool may allow interesting comparisons between different geographical and ethnical populations. It may also allow a thorough investigation of temporal trends in stroke knowledge. As stressed by Sudlow and Warlow (1996), "comparisons are only meaningful if they are based on studies that use similar definitions, methods, and data presentation". Thus, the use of a standard tool facilitates the process of data analysis and enhances the reliability of the findings. This novel tool may be particularly appropriate for health professionals who are in a prime position to evaluate health educational needs and address them. In this view, they can apply this tool to gauge stroke knowledge and use the findings in the planning of educational initiatives in communities.

\section{Declarations}

Author Contributions: Conceptualization, R.G., and P.G.; methodology, R.G., and P.G..; soft-ware, R.G.; validation, R.G., and P.G.; formal analysis, R.G., and P.G.; investigation, R.G.; re-sources, R.G.; data curation, R.G.; writing-original draft preparation, R.G.; writing - review and editing, P.G..; visualization, R.G, and P.G. All authors have read and agreed to this version of the manuscript

Funding: This research received no specific grant from any funding agency in the public, commercial, or not-for-profit sectors.

Institutional Review Board Statement: The study was conducted according to the guidelines of the Declaration of Helsinki and approved by the Institutional Review Board of the University of Sheffield (protocol code 012704; 28/03/2017)

Informed Consent Statement: Informed consent was obtained from all subjects involved in the study

Data Availability Statement: The data presented in this study are available on request from the corresponding author.

Conflicts of Interest: The authors declare that there is no conflict of interest 


\section{References}

[1] Billings-Gagliardi, S., \& Mazor, K. M. (2005). Development and validation of the stroke action test. Stroke; a journal of cerebral circulation, 36(5), 1035-1039.

[2] Braun, V., \& Clarke, V. (2006). Using thematic analysis in psychology. Qualitative research in psychology, 3(2), 77-101. DOI: 10.1191/1478088706qp063oa

[3] Centers for Disease Control and Prevention. (2011). Use of a registry to improve acute stroke care -seven states, 2005-2009. MMWR Morbidity \& Mortality Weekly Report, 60(7), 206-210.

[4] Cicchetti, D. V. (1994). Guidelines, criteria, and rules of thumb for evaluating normed and standardized assessment instruments in psychology. Psychological Assessment, 6(4), 284-290.

[5] Creswell, J. W. (2015). A concise introduction to mixed methods research. Thousand Oaks, California: SAGE.

[6] DeVellis, R. F. (2012). Scale development: theory and applications. Thousand Oaks, California: SAGE.

[7] Fitzpatrick, R., Davey, C., Buxton, M., \& Jones, D. (1998). Evaluating patient-based outcome measures for use in clinical trials. Health Technology Assessment, 2(14), 1-74.

[8] Grech, P., \& Grech, R. (2021). Stroke knowledge: Developing a framework for data integration in a sequential exploratory mixed-method study. Research Methods in Medicine \& Health Sciences 2 (2), 68-81. DOI: 10.1177/2632084320978040

[9] Haladyna, T. M. (2004). Developing and validating multiple-choice test items (3rd ed. ed.): Mahwah, N.J.: Lawrence Erlbaum Associates.

[10] Haynes, S., Richard, D., \& Kubany, E. (1995). Content Validity in Psychological Assessment: A Functional Approach to Concepts and Methods (Vol. 7).

[11] Hou, W.-H., Hoffmann, T., Huang, Y.-J., Wu, T.-Y., Chen, S.-S., \& Hsieh, C.-L. (2017). A systematic review of tests assessing stroke knowledge. Journal of Cardiovascular Nursing, 32(3), 271-280.

[12] Inzitari, D., Eliasziw, M., Gates, P., Sharpe, B. L., Chan, R. K. T., Meldrum, H. E., \& Barnett, H. J. M. (2000). The causes and risk of stroke in patients with asymptomatic internal carotid artery stenosis. The New England Journal of Medicine, 342(23), 1693-1701. DOI: 10.1056/nejm200006083422302

[13] Jordan, J. E., Osborne, R. H., \& Buchbinder, R. (2011). Critical appraisal of health literacy indices revealed variable underlying constructs, narrow content, and psychometric weaknesses (Vol. 64, pp. 366-379). Elmsford: Elsevier Science Ltd.

[14] Moher, D., Liberati, A., Tetzlaff, J., \& Altman, D. G. (2009). Preferred reporting items for systematic reviews and meta-analyses: the PRISMA statement. Annals of Internal Medicine, 151(4), 264-269.

[15] Mokkink, L. B., Terwee, C. B., Patrick, D. L., Alonso, J., Stratford, P. W., Knol, D. L., . . de Vet, H. C. W. (2010). The COSMIN study reached an international consensus on taxonomy, terminology, and definitions of measurement properties for health-related patient-reported outcomes. Journal of Clinical Epidemiology, 63(7), 737-745. DOI: 10.1016/j.jclinepi.2010.02.006

[16] O'Mahony, P., Rodgers, H., Thomson, R., Dobson, R., \& James, O. (1997). Satisfaction with information and advice received by stroke patients. Clinical Rehabilitation, 11(1), 68-72.

[17] Parahoo, K. (2014). Nursing research: principles, process, and issues (Third ed.). London, UK: Palgrave Macmillan.

[18] Polit, D. F., \& Beck, C. T. (2010). Generalization in quantitative and qualitative research: Myths and strategies. International Journal of Nursing Studies, 47(11), 1451-1458. DOI: 10.1016/j.jinurstu.2010.06.004

[19] Sudlow, C. L. M., \& Warlow, C. P. (1996). Comparing stroke incidence worldwide. What makes studies comparable? Stroke, 27, 550-558. DOI: 10.1161/01.STR.27.3.550

[20] Sullivan, K., \& Dunton, N. (2004). Development and validation of the stroke knowledge test. Topics in Stroke Rehabilitation, 11(3), 19-28.

[21] Tay, L., \& Jebb, A. T. (2017). The SAGE encyclopedia of industrial and organizational psychology (2nd ed.). Newcastle upon Tyne, UK: SAGE.

[22] Thomas, J., \& Harden, A. (2008). Methods for the thematic synthesis of qualitative research in systematic reviews. BMC Medical Research Methodology, 8(1), 45-55. DOI: 10.1186/1471-2288-8-45

[23] Trochim, W. M. K. (2001). Research Methods Knowledge Base: Atomic Dog Pub

\section{Stroke Knowledge Assessment Tool (SKAT)}

Note: This is an online tool and whilst the content remains the same, the format may vary slightly when viewed online.

\section{Section 1: Demographics}

\section{Kindly circle or fill in the most appropriate answer}

\begin{tabular}{|l|l|l|}
\hline D1 & Age & yrs \\
\hline D2 & Gender & $\begin{array}{l}\text { M } \\
\text { F }\end{array}$ \\
\hline D3 & Nationality & $\begin{array}{l}\text { Maltese } \\
\text { Other }\end{array}$ \\
\hline D4 & Ethnicity & \\
\hline D5 & Region of Malta & $\begin{array}{l}\text { Gozo } \\
\text { North } \\
\text { Central }\end{array}$ \\
\hline
\end{tabular}




\begin{tabular}{|l|l|l|}
\hline & & South \\
\hline D6 & Education & $\begin{array}{l}\text { Primary } \\
\text { Secondary } \\
\text { Tertiary }\end{array}$ \\
\hline D7 & Income & $\begin{array}{l}< \\
\text { between } \\
>\end{array}$ \\
\hline D8 & & $\begin{array}{l}\text { Previous stroke } \\
\text { Cardiovascular disease Diabetes } \\
\text { Hypertension } \\
\text { Smoking History }\end{array}$ \\
\hline D9 & Risk Factors & $\begin{array}{l}\text { Y } \\
\text { N }\end{array}$ \\
\hline
\end{tabular}

\section{Section 2: Stroke awareness}

Kindly react to each statement by selecting one of the possible replies from the following scale:

\begin{tabular}{|c|c|c|c|c|}
\hline $\mathbf{1}$ & $\mathbf{2}$ & $\mathbf{3}$ & $\mathbf{4}$ & $\mathbf{5}$ \\
Strongly Agree & Agree & Undecided & Disagree & Strongly Disagree \\
\hline
\end{tabular}

\begin{tabular}{|l|l|l|}
\hline \multicolumn{2}{|l|}{ Answer } \\
\hline Knowledge & & \\
\hline K1 & Smoking is a risk factor for stroke & \\
\hline K2 & Stroke is commoner than breast cancer & \\
\hline K3 & Chest pain is a common symptom of stroke & \\
\hline K4 & Loss of vision and vertigo can be secondary to stroke & \\
\hline K5 & Previous transient ischaemic attacks (TIAs) increase future stroke risk & \\
\hline K6 & Stroke affects the heart & \\
\hline K7 & Difficulty to talk or understand speech should raise suspicion of stroke & \\
\hline K8 & Stroke is a rare cause of long-term disability & \\
\hline K9 & Stroke is more common in females & \\
\hline K10 & Diabetes increases the risk of stroke & \\
\hline K11 & Headache may occasionally be secondary to stroke & \\
\hline K12 & Sudden loss of sensation is not related to stroke & \\
\hline K13 & Stroke is always characterised by symptoms & \\
\hline K14 & Muscle or limb power is not affected in stroke & \\
\hline K15 & $\begin{array}{l}\text { The acronym 'FAST' in the 'Act FAST' campaign stands for Face, Arms, } \\
\text { Speech and Time }\end{array}$ & \\
\hline K16 & $\begin{array}{l}\text { Patients who have suffered a stroke should be advised to adopt a sedentary } \\
\text { lifestyle }\end{array}$ & \\
\hline K17 & $\begin{array}{l}\text { Mass media adverts and educational leaflets on stroke are effective in } \\
\text { increasing stroke awareness and education }\end{array}$ & \\
\hline Response & & One should only call his GP if his symptoms fail to improve \\
\hline R1 & One should attend a polyclinic or a nearby hospital immediately & \\
\hline R2 & One should act fast and avoid unnecessary delays & \\
\hline R3 & One should discuss with his/the patient's relatives before taking any action & \\
\hline R4 & The emergency phone number is 112 & \\
\hline R5 & Stroke symptoms and long term outcome may improve if the appropriate & \\
\hline R6 & Proatment is given \\
\hline R7 & One should 'wait and see' before taking any action & \\
\hline R8 & The patient should have his lower limbs elevated & \\
\hline R9 & & \\
\hline
\end{tabular}




\begin{tabular}{|c|c|}
\hline R10 & Paracetamol is not an effective treatment against stroke \\
\hline R11 & Stroke will progress no matter one's reactions \\
\hline R12 & One should call for an ambulance immediately \\
\hline R13 & The affected person may not realise that he is suffering a stroke \\
\hline R14 & $\begin{array}{l}\text { If the patient is able to drive to the emergency department, he should do so } \\
\text { immediately }\end{array}$ \\
\hline R15 & 'Clot buster' treatment (t-PA) cannot be administered after 6 hours \\
\hline R16 & $\begin{array}{l}\text { Brain cells are damaged irreversibly in stroke, and the extent of damage is } \\
\text { time-dependent }\end{array}$ \\
\hline R17 & $\begin{array}{l}\text { A stroke sufferer should always call for help himself so as not to be a burden } \\
\text { on others }\end{array}$ \\
\hline
\end{tabular}

